\title{
Mass Quadrupole Spectrometry for Infrared Laser-Generated Plasmas Detection
}

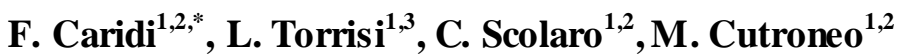 \\ ${ }^{1}$ Dip to di Fisica e Scienze della Terra, Viale F. Stagno d'Alcontres, 31, 98166, Messina, Italy \\ ${ }^{2}$ INFN-Sez. CT, Gruppo coll. di Messina, Viale F. Stagno d'Alcontres, 31, 98166, Messina, Italy \\ ${ }^{3}$ INFN-Laboratori Nazionali del Sud, Via S. Sofia 44, Catania, Italy
}

\begin{abstract}
A nanosecond pulsed Nd:YA G laser, operating at the fundamental wavelength of $1064 \mathrm{~nm}$ and at an intensity of about $10^{10} \mathrm{~W} / \mathrm{cm}^{2}$, was employed to irradiate the $\mathrm{Cu}_{0.4} \mathrm{~W}_{0.6}$ metallic alloy and the relative pure components $(\mathrm{Cu}$ and $\mathrm{W})$ in vacuum to investigate about the ablation. Produced plasmas were characterized in terms of thermal and Coulo mb interactions evaluating the equivalent temperatures and acceleration voltages developed in the non-equilibriu mp lasma core. The particles emission, produced along the normal to the target surface, was investigated by measuring, with the special electrostatic mass quadrupole spectrometer Hiden EQP 300, neutral and ion energy distributions and fitting experimental data with the "Coulo mb-Boltzmann-shifted" function. Results indicated that the metal alloy stoichiometry, in a first approximation, is well transported to the ion and neutral stoichiometry of the plas ma.
\end{abstract}

Keywords $\mathrm{Cu}-\mathrm{W}$ Alloy, Infrared Laser-Generated Plasma, Neutral, Ion Energy Distributions, Mass Quadrupole Spectrometry

\section{Introduction}

Intense pulsed laser beams focused on a solid material produce ablation and formation of hot non-equilibrium plasmas, which have been used for several applications in these last years, such as deposition of thin films, generation of ions at high energy and charge state, treatment of surfaces, ion implantation, medical applications, etc.[1, 2].

Energetic plas ma ions permit to induce ion implantation effects in the exposed surfaces, improving the film-substrate adhesion, to induce activation and growth of nanostructures, such as carbon nanotubes and fullerenes[3]. Energetic neutral emission determines the fractional ionization of the plasma and play an important role in the plas ma properties (temperature, density, ion neutralization, charge exchange, ionization processes, etc.).

Processes developed inside the laser-generated plasma are very complex and depend on many different parameters, such as the laser intensity, target composition, irradiation conditions, etc. Thus, for their description, many effects, such as optical absorption, heat conduction, phase transitions, flu id dynamics, inverse Bremmstrahlung and self-focusing, were introduced[4].

Although these plasmas are in non-equilibrium conditions

* Corresponding author:

fcaridi@unime.it (F. Caridi)

Published online at http://journal.sapub.org/ajcmp

Copyright (C) 2012 Scientific \& Academic Publishing. All Rights Reserved and not really thermalized, the concept of "equivalent temperature", referred to ions, electrons and neutrals in near local thermal equilibrium (NLTE) conditions, is employed in order to characterize the plas ma properties, as reported in literature [5].

In this work a $3 \mathrm{~ns} \mathrm{Nd}$ :YA G laser operating in the infrared region with relatively high pulse energy is employed to irradiate copper, tungsten and the alloy of $40 \% \mathrm{Cu}$ and $60 \%$ $\mathrm{W}$, in vacuum. Obtained plas mas are investigated in terms of neutral and ion emission. The aim is to investigate about the ablation, in order to know if the alloy stoichiometry is maintained in the plasma ion and neutral components, in the case of two metals having very different physical and chemical properties.

\section{Materials and Methods}

The employed laser is a Q-switched Nd:Yag pulsed laser, operating at $1064 \mathrm{~nm}$ wavelength, $3 \mathrm{~ns}$ pulse duration, 1-180 $\mathrm{mJ}$ pulse energy, in single pulse or repetition rate $(1-10 \mathrm{~Hz})$ mode. The laser beam is focused through a convergent lens on the solid target placed inside a vacuum chamber at $10^{-6}$ mbar. The optimum focalization distance $(50 \mathrm{~cm})$ was determined minimizing the spot dimension observed on the target. The spot diameter is about $1 \mathrm{~mm}$ and the incidence angle is $45^{\circ}$. This angle can be changed by the operator moving a vacuum feedthrough at which the target holder is fixed. 
The employed targets consist of pure $\mathrm{Cu}$ and $\mathrm{W}$ sheets and an alloy of $40 \% \mathrm{Cu}$ and $60 \% \mathrm{~W}$, with a polished $1 \mathrm{~cm}^{2}$ surface and $1 \mathrm{~mm}$ thickness. They can be moved vertically with the vacuum feedthrough, so that each laser shot can hit a fresh flat surface.

A special electrostatic mass quadrupole spectrometer, the so-called Electrostatic Quadrupole Plasma (EQP Hiden 300), was employed to monitor particles ejected from the plas ma along the target normal surface, with a mass range within 1 and 300 amu. The instrument detects neutrals and charged particles depending on the filament state (with filament "on" neutrals and ions are both detected while with filament "off" only ions are detected). The charge state is monitored through the mass-to-charge ratio measurement performed by an electrostatic ion deflection. It measures the particle energy with an accuracy of $1 \mathrm{eV}$ and plots the energy distribution of neutral and charged species in the energy range $1 \mathrm{eV}-1 \mathrm{keV}$. The spectra acquisition occurs operating at $10 \mathrm{~Hz}$ laser repetition rate, and it is triggered with the laser shot. EQP uses a $45^{\circ}$ electrostatic deflection, in order to measure the ion energy, and a differential pumping at $10^{-7}$ mbar pressure. The instrument consists of four main sections: the ionisation source; the electrostatic energy filter; the mass filter; the secondary electron multiplier (SEM) detector. More details on the EQP instrument are reported in the literature[6].
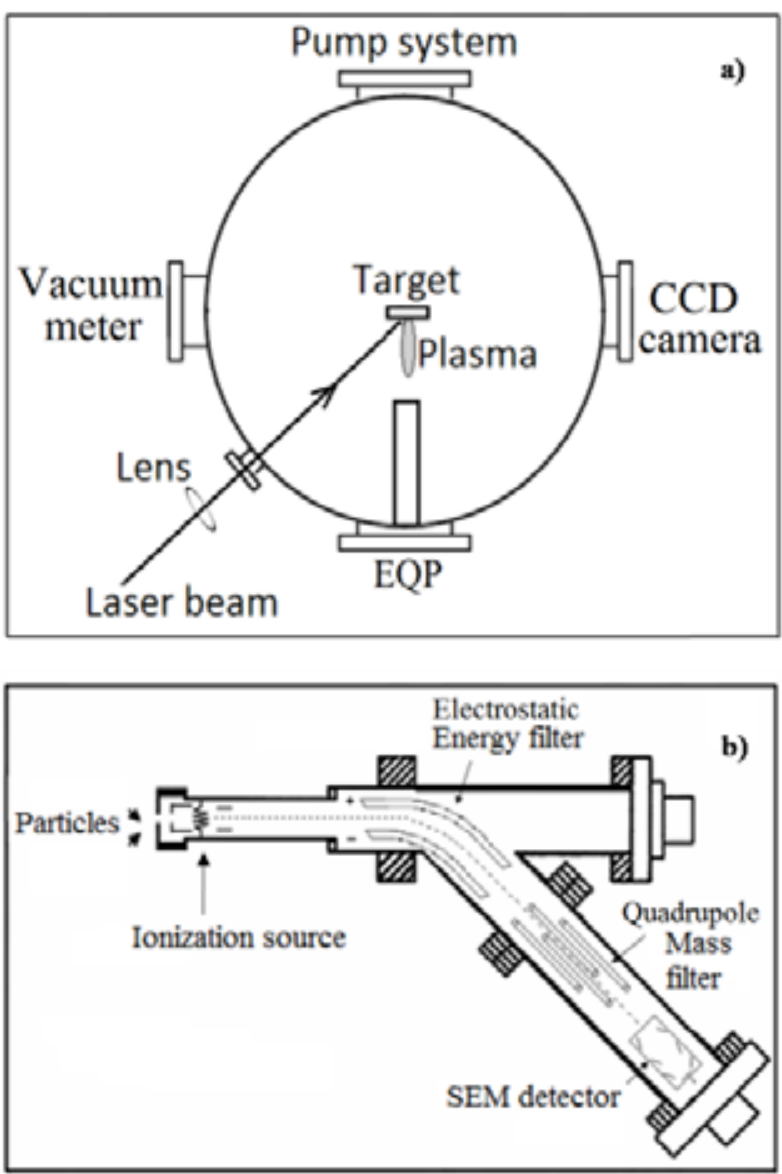

Figure 1. A plot of the experimental setup (a) and a scheme of the EQP detector (b)
Fig. 1 (a) shows a plot of the experimental setup and Fig. 1 (b) reports a scheme of the EQP detector.

EQP spectra were analys ed in order to separate the neutral component from the ionic one and to plot particle energy distributions for various charge states.

The fits of experimental energy distributions were performed with the "Coulomb-Boltzmann-shifted" function[7]:

$$
f(E)=\frac{A}{\sqrt{2 m}} \sqrt{\frac{1}{(\pi k T)^{3}}} \exp \left[-\frac{1}{k T}\left(\sqrt{E}-\sqrt{E_{k}}-\sqrt{E_{C}}\right)^{2}\right](1)
$$

where $A$ represents the normalization constant, $m$ the atomic mass, $E$ the total energy along the normal to the target surface, $E_{K}$ the adiabatic expansion energy in vacuum and $E_{C}$ the Coulomb energy.

The ablation yield was measured off-line by calculating the number of removed atoms per laser pulse from the laser-generated crater on the target. The crater profiles were investigated with the surface profiler (Tencor-P10), which depth and lateral resolution were about $10 \mathrm{~nm}$ and $1 \mu \mathrm{m}$, respectively.

A CCD camera was placed at $90^{\circ}$ with respect to the target normal direction. It was triggered with the laser pulse in order to observe the visible light emitted from the plume, integrating with a variable exposition time.

\section{Results and Discussion}

Figure 2 shows a comparis on of crater profiles obtained irradiating with 50 laser shots, $1 \mathrm{~Hz}$, three employed targets $\left(\mathrm{Cu}, \mathrm{W}\right.$ and $\left.\mathrm{Cu}_{0.4} \mathrm{~W}_{0.6}\right)$ in the same experimental conditions, at $0^{\circ}$ incidence angle and $180 \mathrm{~mJ}$ pulse energy.

The ablation yield was calculated through the crater volume and the target density $\left(\rho_{\mathrm{Cu}}=8.9 \mathrm{~g} / \mathrm{cm}^{3}, \rho_{\mathrm{W}}=19.3\right.$ $\left.\mathrm{g} / \mathrm{cm}^{3}, \quad \rho_{\mathrm{Cu} 0.4 \mathrm{~W} 0.6}=12.75 \mathrm{~g} / \mathrm{cm}^{3}\right)$, giving a value of $2.4 \times 10^{16}$ /pulse and $1.27 \times 10^{16} /$ pulse for $\mathrm{Cu}$ and $\mathrm{W}$, respectively, while for the alloy $\mathrm{Cu}_{0.4} \mathrm{~W}_{0.6}$ an intermediate value of $1.67 \times 10^{16}$ /pulse was obtained.

Figure 3 shows typical EQP spectra obtained ablating $\mathrm{Cu}$ (a) and $\mathrm{Cu}_{0.4} \mathrm{~W}_{0.6}$ (b) targets and detecting copper at $180 \mathrm{~mJ}$ pulse energy, $10 \mathrm{~Hz}$ repetition rate, $45^{\circ}$ incidence angle and $0^{\circ}$ detection angle.

Spectra report the yield signal versus particle energy for neutral and ionized atoms. The fit of experimental data (points) indicates that particles have typical Boltzmann distributions, peaked at different energies depending on their charge state. In particular, $\mathrm{Cu}$ neutrals are peaked at energies of about $35 \mathrm{eV}$ and $46.5 \mathrm{eV}$ for $\mathrm{Cu}$ and $\mathrm{Cu}_{0.4} \mathrm{~W}_{0.6}$ targets, respectively, demonstrating that $\mathrm{Cu}$ particles energies increase in presence of $\mathrm{W}$, due to the higher electron density of the produced plasma. Neutrals energy distributions contain direct information on the plasma temperature. Along the normal to the target irradiation this energy is due to two different contributions: the first is due to the thermal energy, $\mathrm{E}_{\mathrm{T}}=3 \mathrm{kT} / 2$ and the second to the adiabatic expansion energy, $\mathrm{E}_{\mathrm{K}}=\gamma \mathrm{kT} / 2$, where $k$ is the Boltzmann constant, $T$ is the temperature, and $\gamma$ is the adiabatic coefficient (1.67 for 
monoatomic species). The fit of experimental spectra indicates that the temperature for $\mathrm{Cu}$ and $\mathrm{Cu}_{0.4} \mathrm{~W}_{0.6}$ plasmas is $15 \mathrm{eV}$ and $20 \mathrm{eV}$, respectively, demonstrating that it increases in presence of $\mathrm{W}$ due to the increase of the plasma electron density. Ion energy distributions contain information not only about the temperature but also about Coulomb interactions. In this case, in fact, a third contribution is involved in the total ion energy; this is the Coulomb energy, $E_{C}=z_{0} V_{0}$, where ze is the ion charge and $\mathrm{V}_{0}$ is the equivalent acceleration voltage developed inside the non-equilibrium plasma. First ionized $\mathrm{Cu}$ atoms are peaked at about $112 \mathrm{eV}$ and $136 \mathrm{eV}$ for $\mathrm{Cu}$ and $\mathrm{Cu}_{0.4} \mathrm{~W}_{0.6}$ plasmas. $\mathrm{Cu}$ ions with higher charge states have a regular energy shift of about $75 \mathrm{eV}$ and $90 \mathrm{eV}$ for $\mathrm{Cu}$ and $\mathrm{Cu}_{0.4} \mathrm{~W}_{0.6}$ targets, respectively, for a total number of 4 and 8 charge states, respectively. All experimental data are reported in Table 1.
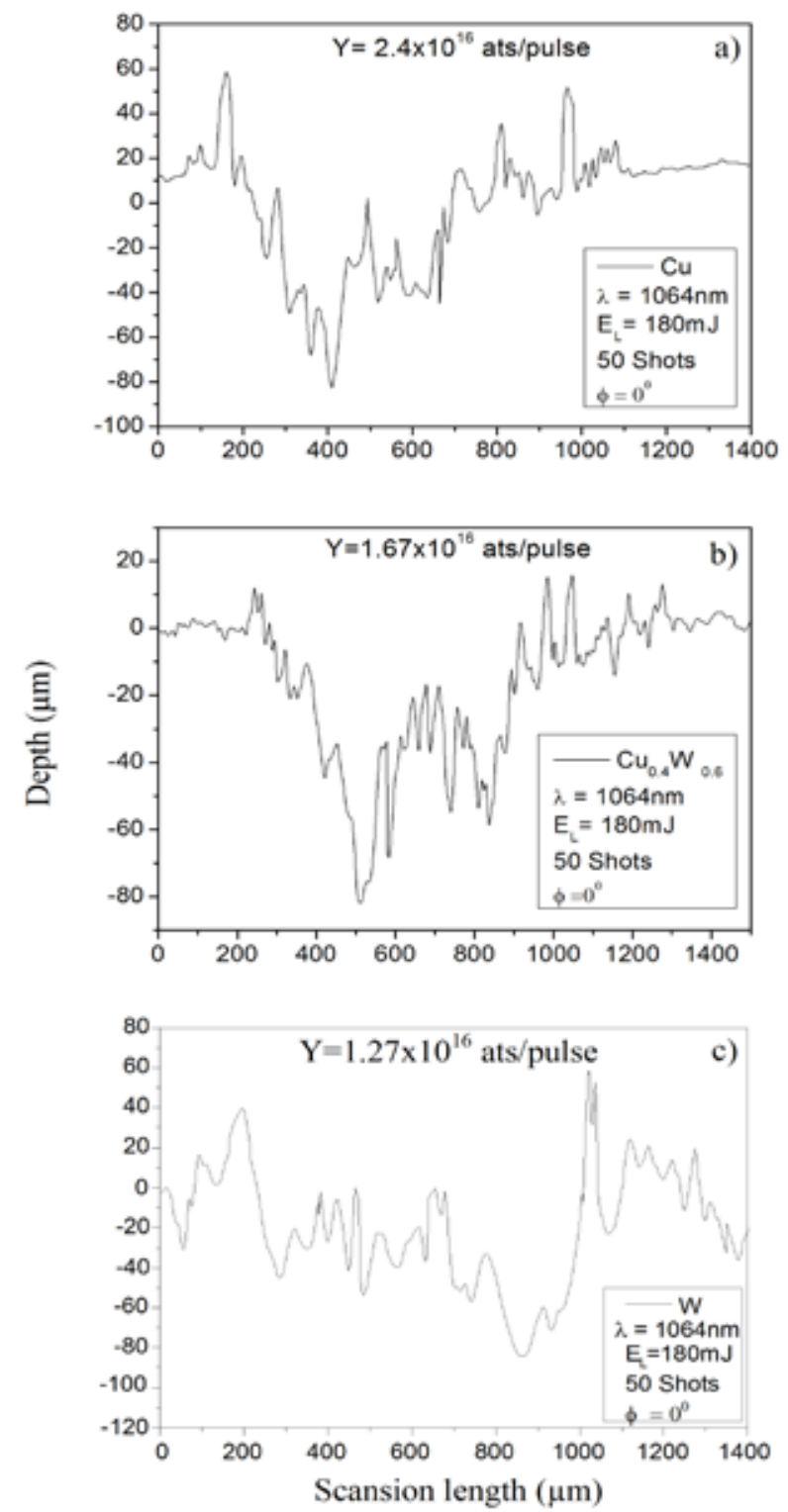

Figure 2. A comparison of crater profiles obtained irradiating with 50 laser shotsthree employed targets $\left(\mathrm{Cu}, \mathrm{Cu}_{.4} \mathrm{~W}_{0.6}\right.$ and W $)$

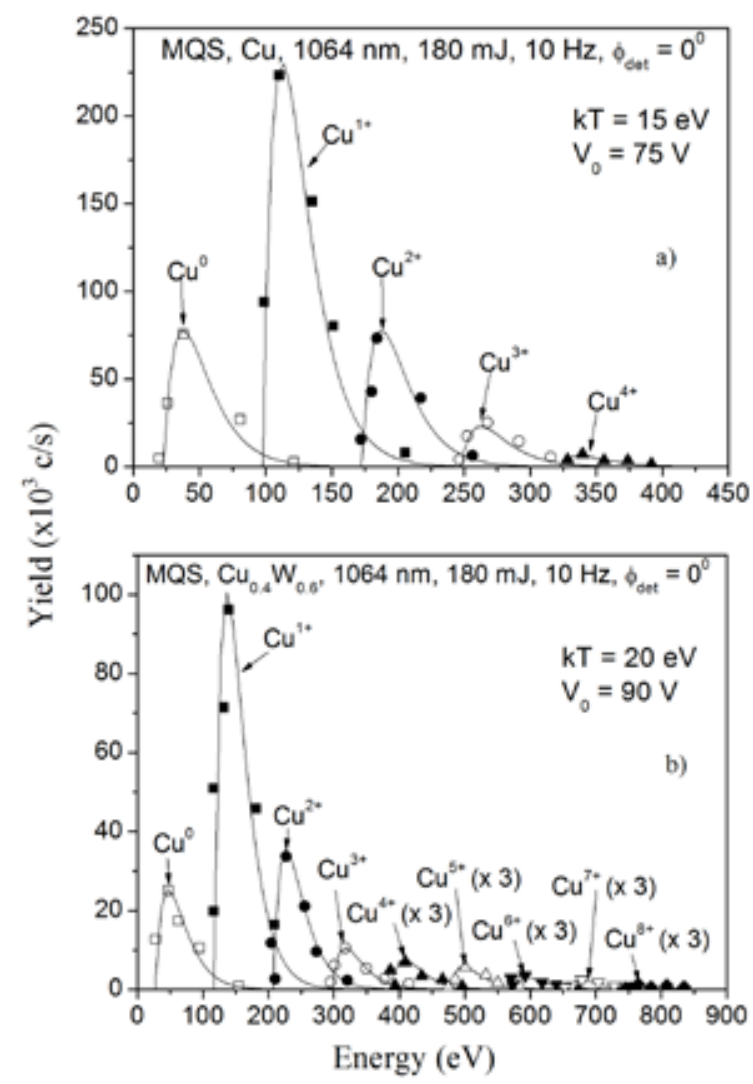

Figure 3. Typical EQP spectra obtained ablating $\mathrm{Cu}(\mathrm{a})$ and $\mathrm{Cu}_{0.4} \mathrm{~W}_{0.6}$ (b) targets and detecting copper

Table 1. Ion charge states and peak energy, plasma fractional ionization, equivalent temperat ure and acceleration voltage for all irradiat ed targets

\begin{tabular}{|c|c|c|c|}
\hline \multicolumn{4}{|c|}{ Cu target } \\
\hline & Peak Energy $(\mathrm{eV})$ & $\mathrm{kT}(\mathrm{eV})$ & $\mathrm{V}_{0}(\mathrm{~V})$ \\
\hline $\mathrm{Cu}^{0}$ & 35 & \multirow{5}{*}{15} & \multirow{5}{*}{75} \\
\hline $\mathrm{Cu}^{1+}$ & 112 & & \\
\hline $\mathrm{Cu}^{2+}$ & 189 & & \\
\hline $\mathrm{Cu}^{3+}$ & 260 & & \\
\hline $\mathrm{Cu}^{4+}$ & 340 & & \\
\hline \multicolumn{4}{|c|}{ W target } \\
\hline & Peak Energy $(\mathrm{eV})$ & $\mathrm{kT}(\mathrm{eV})$ & $\mathrm{V}_{0}(\mathrm{~V})$ \\
\hline $\mathrm{W}^{0}$ & 81 & \multirow{8}{*}{35} & \multirow{8}{*}{115} \\
\hline $\mathrm{W}^{1+}$ & 195 & & \\
\hline $\mathrm{W}^{2+}$ & 315 & & \\
\hline $\mathrm{W}^{3+}$ & 420 & & \\
\hline $\mathrm{W}^{4+}$ & 540 & & \\
\hline$\overline{W^{5+}}$ & 645 & & \\
\hline $\mathrm{W}^{6+}$ & 770 & & \\
\hline $\mathrm{W}^{7+}$ & 880 & & \\
\hline \multicolumn{4}{|c|}{$\mathrm{Cu}_{0.4} \mathrm{~W}_{0.6}$ target } \\
\hline & Peak Energy (eV) & $\mathrm{kT}(\mathrm{eV})$ & $\mathrm{V}_{0}(\mathrm{~V})$ \\
\hline $\mathrm{Cu}^{0}, \mathrm{~W}^{0}$ & 46.5 & \multirow{10}{*}{20} & \multirow{10}{*}{90} \\
\hline $\mathrm{Cu}^{1+}, \mathrm{W}^{1+}$ & 136 & & \\
\hline $\mathrm{Cu}^{2+}, \mathrm{W}^{2+}$ & 227 & & \\
\hline $\mathrm{Cu}^{3+}, \mathrm{W}^{3+}$ & 315 & & \\
\hline $\mathrm{Cu}^{4+}, \mathrm{W}^{4+}$ & 405 & & \\
\hline $\mathrm{Cu}^{5+}, \mathrm{W}^{5+}$ & 500 & & \\
\hline $\mathrm{Cu}^{6+}, \mathrm{W}^{6+}$ & 585 & & \\
\hline $\mathrm{Cu}^{7+}, \mathrm{W}^{7+}$ & 675 & & \\
\hline $\mathrm{Cu}^{8+}, \mathrm{W}^{8+}$ & 766 & & \\
\hline $\mathrm{W}^{9+}$ & 855 & & \\
\hline
\end{tabular}


Figure 4 shows typical EQP spectra obtained ablating $\mathrm{W}$ (a) and $\mathrm{Cu}_{0.4} \mathrm{~W}_{0.6}$ (b) targets and detecting tungsten at the same experimental conditions as before.

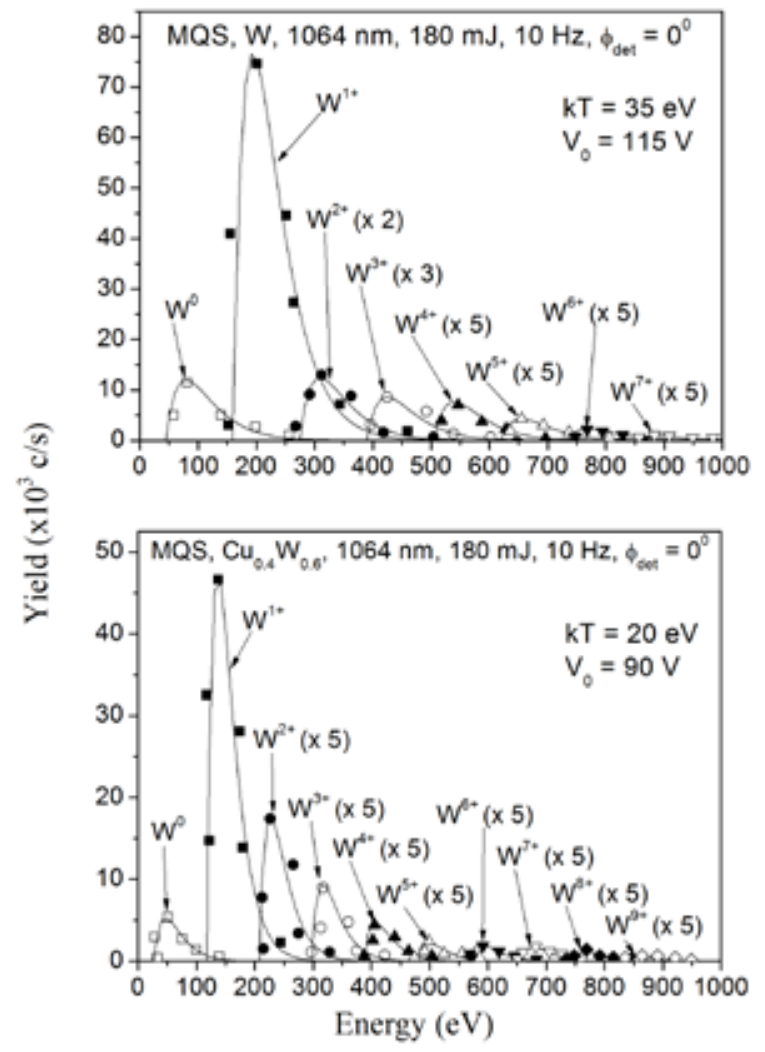

Figure 4. Typical EQP spectra obtained ablating W (a) and $\mathrm{Cu}_{0.4} \mathrm{~W}_{0.6}$ (b) targets and detecting tungsten

In this case, $\mathrm{W}$ neutrals are peaked at energies of about 81 $\mathrm{eV}$ and $46.5 \mathrm{eV}$ for $\mathrm{W}$ and $\mathrm{Cu}_{0.4} \mathrm{~W}_{0.6}$ targets, respectively, while first ionized $\mathrm{W}$ ato $\mathrm{ms}$ are peaked at about $195 \mathrm{eV}$ and $136 \mathrm{eV}$ in the two cases, demonstrating that the $\mathrm{W}$ ion energy decreases for the $\mathrm{Cu}_{0.4} \mathrm{~W}_{0.6}$ alloy due to the lower plas ma electron density. $\mathrm{W}$ ions with higher charge states have a regular energy shift of about $115 \mathrm{eV}$ and $90 \mathrm{eV}$ for $\mathrm{W}$ and $\mathrm{Cu}_{0.4} \mathrm{~W}_{0.6}$ targets, respectively, for a total number of 7 and 9 charge states, respectively. The fit of experimental spectra indicates that the temperature for $\mathrm{W}$ and $\mathrm{Cu}_{0.4} \mathrm{~W}_{0.6}$ plasmas is $35 \mathrm{eV}$ and $20 \mathrm{eV}$, respectively. Experimental data are summarized in Tab. I.

Different results obtained irradiating employed targets under the same experimental conditions can be explained on the basis of different physical properties of elements.

One of these properties is represented by the density of electrons of conduction in metals, which are weakly bounded to the elemental atoms. In general, for elements characterized by an high electron density value, there will be a higher amount of electrons in the dense vapor, immed iately after the laser ablation. These electrons absorb the laser photons energy through the inverse bremsstrahlung process reaching energies suitable to induce ionization processes.

Another important physical parameter is the ablation threshold, calculated through the approach given by Torrisi et al.[8]. For copper, that has a lower ablation threshold (1.04 $\mathrm{J} / \mathrm{cm}^{2}$ ) and a low boiling point, only a small fraction of the laser pulse energy is used for the evaporation. As a consequence, above the threshold the ablation yield is generally high, because of the remaining high laser pulse energy transferred to the plasma. On the contrary, for tungsten, with a higher value of the ablation threshold (1.3 $\mathrm{J} / \mathrm{cm}^{2}$ ) and a high boiling point, a higher a mount of the laser pulse energy is necessary to produce its evaporation in vacuum. The ablation yield is lower as a consequence of the remaining low pulse energy transferred to the plasma. Moreover, we expect that the mean energy transferred by the laser pulse to the atoms of the vapor is low when the target has a low evaporation point and high ab lation yield, as $\mathrm{Cu}$ for example, because many atoms compose a dense vapor[9].

On the contrary, the mean energy is high when the target has a high evaporation point and low ablation yield, as $\mathrm{W}$ for example, because the vapor is less dense. This is in good agreement with our experimental results detailed in Table 1.

Results obtained with EQP indicate that the maximum charge state for the ablated targets obtainable at $180 \mathrm{~mJ}$ pulse energy is $4+$ and $8+$ for copper in $\mathrm{Cu}$ and $\mathrm{Cu}_{0.4} \mathrm{~W}_{0.6}$ plasmas, respectively, and 7+ and 9+ for tungsten in $\mathrm{W}$ and $\mathrm{Cu}_{0.4} \mathrm{~W}_{0.6}$ plasmas, respectively.

This result is in good agreement with the ionization potential values of the elements[10].

In fact, assuming for example an average plasma temperature of about $15 \mathrm{eV}$ for copper in $\mathrm{Cu}$ plas ma, the mean thermal electron energy is about $23 \mathrm{eV}$.

Because the energy distribution has a tail up to about three times than the mean energy, this means that electrons with energies of about $76 \mathrm{eV}$ are present in the plasma. At this energy level the expected ionization is $4+$, as reported in Figure 5.

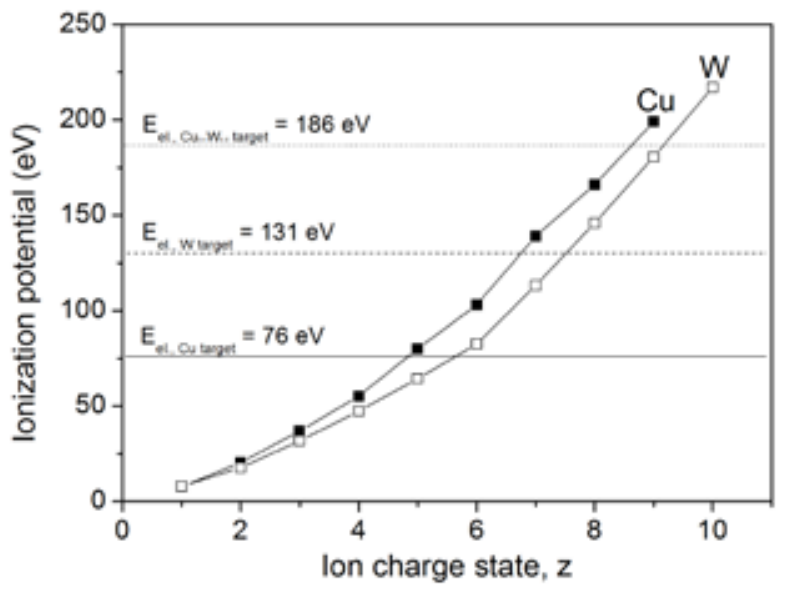

Figure 5. The ionization potential as a function of the ion charge state for the ablat ed targets

Likewise the expected ionization is 7+ for tungsten in $\mathrm{W}$ plasma, 8+ for copper and 9+ for tungsten in $\mathrm{Cu}_{0.4} \mathrm{~W}_{0.6}$ plasma, respectively.

EQP data were analyzed as energy distributions through fits that have been performed taking in consideration that the 
used ion charge state distributions should be in agreement with the trend of the electron ionization cross section.

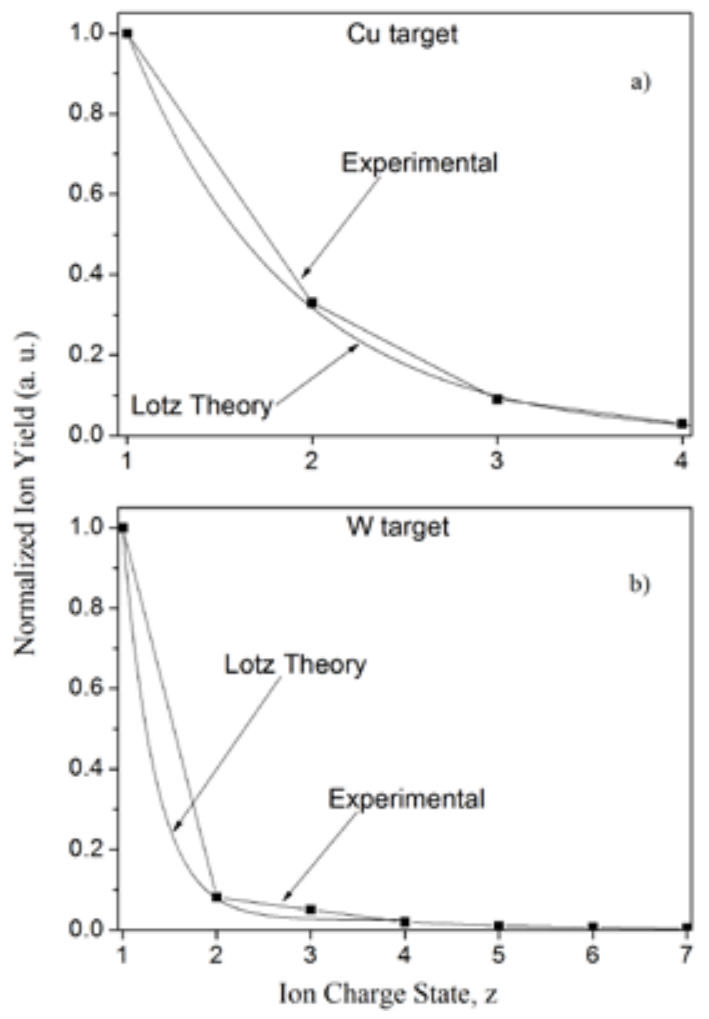

Figure 6. The comparison for EQP ion data elaboration for $\mathrm{Cu}(\mathrm{a})$ and $\mathrm{W}$ (b) targets, respectively

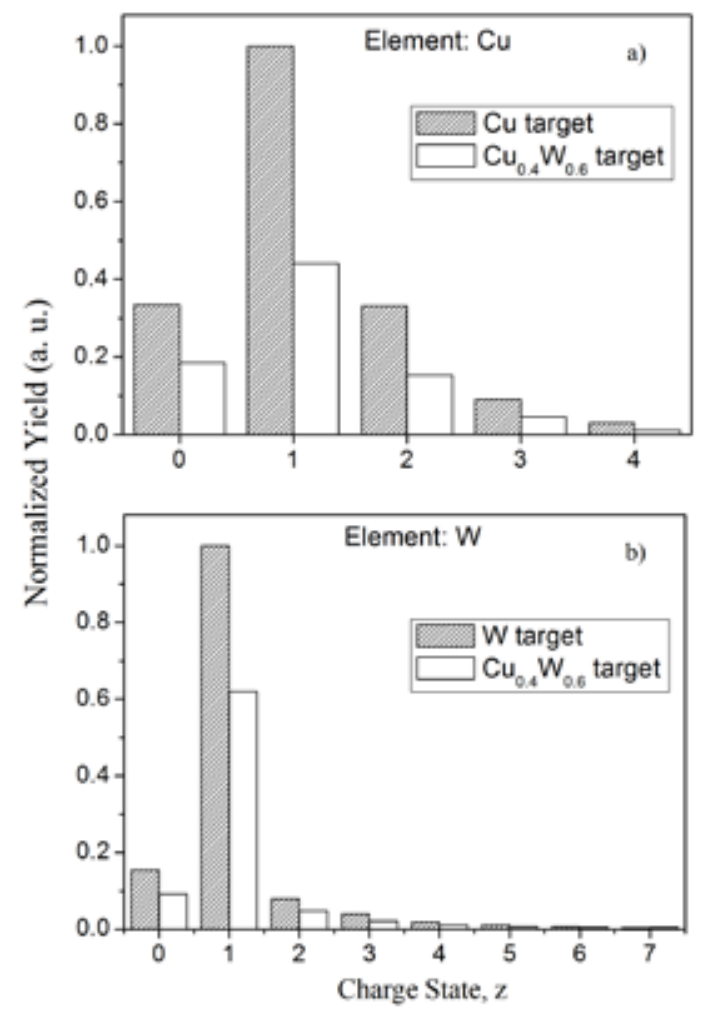

Figure 7. A comparison between thenormalized neutral and ion yields for copper (a) in $\mathrm{Cu}$ and $\mathrm{Cu}_{0.4} \mathrm{~W}_{0.6}$ targets, respectively, and for tungsten (b) in $\mathrm{W}$ and $\mathrm{Cu}_{0.4} \mathrm{~W}_{0.6}$ target s, respect ively
To this reason comparisons between normalized ion charge state distributions and ionization cross-sections based on Lotz theory[11] have been performed.

The cross-sections were calculated using the mean electron energy, $\mathrm{E}_{\mathrm{e}}$, given by the best temperature fit, as reported in Fig. 5.

Fig. 6 shows the comparison for EQP ion data elaboration for $\mathrm{Cu}(\mathrm{a})$ and $\mathrm{W}$ (b) targets irradiation, respectively.

The experimental distributions of the ion yields for the various charge states (squares) and the calculated electron ionization cross section (Lotz theory) show a good agreement.

Fig. 7 shows a co mparis on between the normalized neutral and ion yields for copper (a) in $\mathrm{Cu}$ and $\mathrm{Cu}_{0.4} \mathrm{~W}_{0.6}$ plasmas, respectively, and for tungsten (b) in $\mathrm{W}$ and $\mathrm{Cu}_{0.4} \mathrm{~W}_{0.6}$ plasmas, respectively.

Experimental results follow the stoichiometry of emp loyed samples.

Thus results indicate that the metal alloy stoichiometry, in a first approximation, is well transported to the ion and neutral stoichiometry of the plasma.

\section{Conclusions}

The laser ablation of $\mathrm{Cu}, \mathrm{W}$ and $\mathrm{Cu}_{0.4} \mathrm{~W}_{0.6}$ targets at 1064 $\mathrm{nm}$ was investigated by using an electrostatic deflector mass quadrupole spectrometer. At a laser intensity of about $10^{10}$ $\mathrm{W} / \mathrm{cm}^{2}$ energy spectra show "Coulo mb-Boltzmann-shifted" distributions for various ion charge states. The total energy has three components: thermal, adiabatic and Coulomb.

The neutral monoatomic species characterize the mean plasma temperature, which is about $20 \mathrm{eV}$ at the maximum fluence.

The energy shift of ion energy distributions gives a measure of the equivalent acceleration potential generated inside the plasma during the laser pulse irradiation. The corresponding electrical field, for distances comparable with the Debye length (about $100 \mathrm{~nm}$ ) and for an electron density of the order of $19 \times 10^{16} / \mathrm{cm}^{3}$ can be calculated as $\mathrm{E}=$ $\mathrm{V}_{0} / \lambda_{\mathrm{D}}$ [13]. A maximum value of about $11.5 \mathrm{MV} / \mathrm{cm}$ can be given as a first approximation.

The comparison of our results with literature data is good, confirming the valid ity of our experimental approaches. The original alloy stoichiometry of $40 \% \mathrm{Cu}$ and $60 \% \mathrm{~W}$ is in first approximation found also in the plasma neutrals and ions.

\section{ACKNOWLEDGEMENTS}

The authors gratefully acknowledge useful technical support from Mr. D. Bonanno of the Physics Department of Messina University.

\section{REFERENCES}


[1] R.K. Singh, J. Narayan, Phys. Rev. B 41 8843, 1990.

[2] L. Torrisi, S. Gammino, A.M. Mezzasalma, J. Badziak, P. Parys, J. Wolowski, E. Woryna, L. Laska, M. Pfeifer, K. Rohlena, F.P. Boody, Appl. Surf. Sci. 217319

[3] A. Mangione, L. Torrisi, A. Picciotto, F. Caridi, D. Margarone, E. Fazio, A. La Mantia, and G. Di Marco, Rad. Eff. and Def. in Solids 160, 655 (2005).

[4] D. Giulietti, L.A. Gizzi, Riv. Nuovo Cimento 21 1-93(1998).

[5] L. Torrisi, F. Caridi, A. Picciotto, D. Margarone, A. Borrielli, J. Appl. Phys. 100093306 (2006).

[6] Online available: http://www.hidenanalytical.com/products .

[7] F. Caridi, L. Torrisi, L. Andò, D. Margarone, A. Borrielli, Nucl. Instr. And Meth. B 266 4816-4821 (2008).
[8] L. Torrisi, A. Borrielli, D. Margarone, Nucl. Instr. \& Meth. Phys. Res. Section B 255, 373-379 (2007).

[9] L. Torrisi, S. Gammino, L. Andò, V. Nassisi, D. Doria, A. Pedone, Appl. Surf. Sci. 210, 262-273 (2003).

[10] G. Zschornak, G. Musiol, W. Wagner, Berlin: Akademie der Wissenschaften der DDR., 160-255 (1986).

[11] L. Torrisi, D. Margarone, A. Borrielli, F. Caridi, Appl. Surf. Sci. 254 4007- 4012 (2008).

[12] Y. Tao, Y. Shan, N.Wang, Y. Li, X. Tang, H. Zhang, in: Proceedings of 5th Work on Fast Ignition of Fusion Targets, Funchal, June 2001.

[13] L. Torrisi, F. Caridi, D. Margarone, A. Picciotto, A. Mangione, J.J. Beltrano, Appl. Surf. Sci. 252 6383-6389 (2006).- 